Check for updates

Cite this: RSC Adv., 2018, 8, 14967

Received 23rd January 2018

Accepted 17th March 2018

DOI: $10.1039 / c 8 r a 00675 j$

rsc.li/rsc-advances

\title{
Hypoglycemic effect and mechanism of isoquercitrin as an inhibitor of dipeptidyl peptidase-4 in type 2 diabetic mice
}

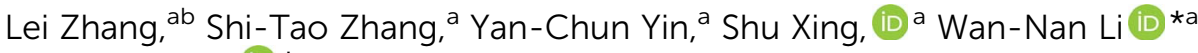 \\ and Xue-Qi Fu iD *a
}

\begin{abstract}
Glucagon-like peptide (GLP)-1 is a potent glucose-dependent insulinotropic gut hormone released from intestinal $L$ cells. The aim of this study was to investigate isoquercitrin as an inhibitor of dipeptidyl peptidase IV (DPP-IV) and determine whether it affects GLP-1 release in normal mice and $\mathrm{NCl}-\mathrm{H} 716$ cells. In vitro, we used chromogenic substrate method detection methods to measure DPP-IV. We found that isoquercitrin was a competitive inhibitor, with $I_{50}$ and $K_{\mathrm{i}}$ values of 96.8 and $236 \mu \mathrm{M}$, respectively. Isoquercitrin and sitagliptin also stimulated GLP-1 release in $\mathrm{NCl}-\mathrm{H} 716$ cells. In vivo, a type 2 diabetic mouse model was established, and oral treatment with different concentration of isoquercitrin and sitagliptin for 8 weeks significantly decreased the fasting blood glucose level. The weight and the levels of serum GLP-1 and insulin of the mice in the isoquercitrin group were higher than those in the model group $(P<0.001)$. An oral glucose tolerance test showed that the isoquercitrin significantly inhibited postprandial blood glucose changes in a dose-dependent manner. These findings demonstrated the hypoglycemic effects of isoquercitrin and indicated that isoquercitrin improved insulin sensitivity by targeting DPP-IV.
\end{abstract}

\section{Introduction}

Diabetes is a worldwide epidemic, with the World Health Organization estimating that more than 220 million people have diabetes worldwide, with greater than $90 \%$ of those having type 2 diabetes mellitus (T2DM). T2DM is thought to develop as a combination of insulin resistance and pancreatic $\beta$-cell failure. ${ }^{1}$ Therefore, identification of novel treatments that would increase pancreatic insulin secretion while protecting pancreatic $\beta$-cells is of great interest. Incretin hormones, such as glucagon-like peptide-1 (GLP-1), are secreted from cells in the gastrointestinal (GI) tract into the circulation in response to nutrient absorption. They are a major component of the mechanism regulating post-prandial insulin secretion when insulin is needed following meals. ${ }^{2}$ However, GLP-1 is rapidly inactivated in vivo by circulating peptidases, mainly dipeptidyl peptidase 4 (DPP-IV), so that they cannot be used for therapeutic purposes. DPP-IV inhibitors provide clinical benefits in patients with T2DM by increasing the levels of glucose-lowering incretin hormones, such as GLP-1. Therefore, the aim of this analysis was to comprehensively assess the pre-clinical efficacy of DPP-IV inhibitors in Chinese T2DM patients and to evaluate

${ }^{a}$ Edmond H. Fischer Signal Transduction Laboratory, School of Life Sciences, Jilin University, Changchun 130012, China

${ }^{b}$ College of Life Science and Technology, Mudanjiang Normal University, Mudanjiang 157011, China whether the response to treatment varies with different types of DPP-IV inhibitors in such patients. ${ }^{3}$

Studies found that flavonoids have an obvious function in modulate DPP4 activity, such as grape seed-derived procyanidins and Pilea microphylla. ${ }^{4,5}$ Isoquercitrin is one of the major bioactive constituents of the apocynum and folium mori, and it widely exists in medicinal plants, food and beverage, fruits and vegetables. Isoquercitrin was shown to be the pharmacologically effective component with potent antioxidant, antiinflammatory, antidepressant, antihypertensive, and lipidlowering activities. ${ }^{6,7}$ In this study, a possible hyperglycaemic mechanism was investigated through an in vitro enzymatic reaction kinetics experiment. We also explored the strength of the hypoglycemic effect and attempted to better understand its mechanisms using cell experiments and a T2DM mouse model.

\section{Materials and methods}

Recombinant E. coli containing the $\Delta$ DPP-IV plasmid was purchased from Beijing Yiqiaoshenzhou Biotechnology Company. A GLP-1 ELISA Kit and an insulin ELISA Kit were purchased from RD Company. All types of restriction endonucleases, gel extraction kits, Plasmid Miniprep kits and T4 DNA ligases were purchased from Fermentas. Triton-100, PEP and BZM were purchased from Sigma-Aldrich. Gly-Pro-PNA was purchased from Roche. NCI-H716 cells were purchased from ATCC America, and all cell culture reagents were from Hyclone. 
Mice with body weights of approximately $20 \pm 2 \mathrm{~g}$ were purchased from the experimental animal center of Jilin University; isoquercitrin and sitagliptin were purchased from Aladdin (Xi'an, People's Republic of China). The kits for the analysis of total triglyceride (TG) and total cholesterol (TC) levels were purchased from Beijing BHKT. STZ, Tris and EDTA were purchased from Beijing DingGuo Biotechnology Company. A Blood Glucose Kit was purchased from Takala.

\section{Preparation of $\triangle D P P-I V$}

The cDNA was obtained with the PMD18 template, which encodes the catalytic domain of DPP-IV ( $\triangle$ DPP-IV); synthesis was performed with polyadenylated mRNA, and the recombinant plasmid was transformed into E. coli Rosstta DE3 plysS and induced by IPTG. The catalytic domain of DPP-IV was amplified by PCR, and then the truncated enzyme was purified by a GST Resin column.

\section{$\Delta$ DPP-IV inhibition assays}

The DPP-IV activities were measured by the addition of $10 \mu \mathrm{L}$ of $10 \mathrm{mmol} \mathrm{L}^{-1}$ Gly-Pro-PNA (the substrate) to a buffer solution ( $\mathrm{pH}$ 8.0) containing $0.1 \mathrm{~mol} \mathrm{~L}^{-1} \mathrm{NaCl}, 1 \mathrm{mmol} \mathrm{L}^{-1}$ ethylene diamine tetra-acetic acid (EDTA), $10 \mu \mathrm{L}$ and $50 \mathrm{ng}$ DPP-IV and recombinant DPP-IV, respectively, along with or without different concentrations of isoquercitrin diluted in dimethyl sulfoxide (DMSO). After incubation for $10 \mathrm{~min}$ at $37{ }^{\circ} \mathrm{C}$, the reactions were terminated with $0.1 \mathrm{~mol} \mathrm{~L}^{-1} \mathrm{NaHCO}_{3}$, and the amount of the product, pentose nucleic acid (PNA), was measured by UV absorbance at a wavelength of $405 \mathrm{~nm}$. The inhibitory potency of inhibitors was evaluated by $\mathrm{IC}_{50}$ values.

\section{Determination of inhibition kinetics}

Substrate (Gly-Pro-PNA) at different concentrations was added separately to the reaction mixtures containing various concentrations of isoquercitrin. The absorbance at $405 \mathrm{~nm}$ was measured to determine the amount of PNA produced. The inhibition kinetic analysis was carried out using the Line weaver-Burk plot of $1 / v$ versus $1 /[\mathrm{S}]$.

\section{Cell culture and stimulation}

NCI-H716 cells were cultured in 1640 medium supplemented with $10 \%$ foetal bovine serum (FBS) at $37{ }^{\circ} \mathrm{C}$ in a humidified atmosphere with $5 \% \mathrm{CO}_{2}$. NCI-H716 cells were incubated with various concentrations of isoquercitrin (dissolved in DMSO) for $6 \mathrm{~h}, 24 \mathrm{~h}, 48 \mathrm{~h}$ and $72 \mathrm{~h}$. Sitagliptin was used in the positive control group. Our pre-experiments involved the use of a series of sitagliptin concentrations $\left(10 \mu \mathrm{mol} \mathrm{L}^{-1}, 50 \mu \mathrm{mol} \mathrm{L}^{-1}\right.$ and 100 $\mu \mathrm{mol} \mathrm{L}{ }^{-1}$ ) for $6 \mathrm{~h}, 24 \mathrm{~h}, 48 \mathrm{~h}$ and $72 \mathrm{~h}$. For the GLP-1 detection, cells were evaluated using a GLP-1 ELISA kit; the UV absorbance was then measured at a wavelength of $450 \mathrm{~nm}$.

\section{Establishment of type 2 diabetic mouse model and treatment protocol}

Ninety male Chinese Kunming mice, which were 8 weeks old and weighed 18-22 g, were used in the studies. All animal trial procedures instituted by the Ethical Committee for the Experimental Use of Animals and for Drug Safety Evaluation were followed. All 90 mice were housed five to a cage in a $12: 12 \mathrm{~h}$ light/dark cycle at an ambient temperature of $22{ }^{\circ} \mathrm{C}-25{ }^{\circ} \mathrm{C}$. For the normal group, 10 mice were fed ordinary chow, and the other 80 mice were fed a high-fat diet (composed of $20 \%$ sucrose, $10 \%$ pork lard, $2.5 \%$ cholesterol, $1 \%$ sodium cholate, and $66.5 \%$ ordinary chow) to induce type 2 diabetes. After 4 weeks, 80 mice were fasted for $8 \mathrm{~h}$ with free access to water and then intraperitoneally injected with STZ $\left(35 \mathrm{mg} \mathrm{kg} \mathrm{kg}^{-1}\right.$ in $0.1 \mathrm{~mol} \mathrm{~L}^{-1}$ citrate-buffered saline, $\mathrm{pH} 4.4$; injection for 3 days, and one injection per day) to induce type 2 diabetes. The STZtreated mice had free access to high-fat food and water. After 1 week, the STZ-treated mice had a fasting blood glucose (FBG) level of $11.1 \mathrm{mmol} \mathrm{L}^{-1}$. These type 2 diabetic mice were separated into five groups (sixteen mice per group), including a diabetes model group that received $0.9 \%$ saline [vehicle], an isoquercitrin low-dose group that received $20 \mathrm{mg} \mathrm{kg} \mathrm{kg}^{-1}$, isoquercitrin, a medium-dose group that received $40 \mathrm{mg} \mathrm{kg}^{-1}$, and an isoquercitrin high-dose group that received a $80 \mathrm{mg} \mathrm{kg}^{-1}$ dose of isoquercitrin (isoquercitrin was dissolved in dimethyl sulfoxide [DMSO] solvent; the sitagliptin group received $20 \mathrm{mg}$ $\mathrm{kg}^{-1}$ doses of sitagliptin). The normal group received $0.9 \%$ saline (vehicle). All the drug stock solutions were diluted in $0.9 \%$ saline and administered through oral gavage once per day for 8 weeks.

\section{Measurement of fasting blood glucose, oral glucose tolerance test (OGTT) results, body weight, cholestenone and lipid profile}

During the treatment period, the body weight and blood glucose of 8 h-fasted mice were measured every week. FBG was measured using a One Touch Ultra Easy glucose reader. Blood samples were obtained from the tail vein of mice. The total TC and TG in serum were measured following the recommended instructions in the commercial kits.

\section{Measurement of GLP-1 and insulin}

After the treatment period, the mice were fasted for $8 \mathrm{~h}$ with free access to water and then received $2 \mathrm{~g} \mathrm{~kg}^{-1}$ doses of glucose. Blood samples were obtained from the tail vein of mice at different times (10 $\mathrm{min}, 20 \mathrm{~min}, 30 \mathrm{~min}, 1 \mathrm{~h}$ and $2 \mathrm{~h}$ ). The samples were held for $30 \mathrm{~min}$ and then centrifuged at $5000 \times g$ for $10 \mathrm{~min}$ at $4{ }^{\circ} \mathrm{C}$. Afterward, the supernatant of each sample was collected. The levels of GLP-1 and insulin were measured using GLP-1 and insulin ELISA kits, respectively.

\section{Statistical analysis}

Data are presented as the mean \pm SD. Statistical analysis was conducted using Student's $t$-test or one-way ANOVA with GraphPad Prism 5 software. A probability value of $P<0.05$ was considered statistically significant. 


\section{Results}

\section{Expression and purification of DPP-IV}

The expression of purified DPP-IV is shown in Fig. 1A. The specific activity was approximately 19500 units per mg. Analysis of the SDS-polyacrylamide gel electrophoresis grey level indicated that the purity of DPP-IV was over $90 \%$, which met the standard for inhibitor screening. The following experiments were then performed with purified DPP-IV.

\section{Exhibited potent DPP-IV inhibitory activity}

Isoquercitrin had an obvious inhibition effect on DPP-IV from 50 types of monomer compounds. The inhibitory potency of isoquercitrin against DPP-IV was evaluated using the concentration-dependent inhibition curves, as shown in Fig. 1B. Isoquercitrin was identified as a DPP-IV inhibitor, with an $\mathrm{IC}_{50}$ of $96.8 \mu \mathrm{mol} \mathrm{L}{ }^{-1}$. To further determine the inhibition type of isoquercitrin against DPP-IV, Lineweaver-Burk analysis was performed. The Lineweaver-Burk plot had a common intercept for five lines on the $1 / v$ axis as the isoquercitrin concentration increased from 0 to $1000 \mu \mathrm{mol} \mathrm{L}{ }^{-1}$, indicating that isoquercitrin inhibits DPP-IV by competing with the substrate for the enzyme active site (Fig. 1C). The inhibition constant $\left(K_{\mathrm{i}}\right)$ was the inhibition constant of isoquercitrin against DPP-IV, and $K_{\mathrm{i}}$ was determined from the intercept of the $x$-axis; thus, $K_{\mathrm{i}}$ was calculated to be $236 \mu \mathrm{mol} \mathrm{L}^{-1}$ (Fig. 1D).

\section{Effects of isoquercitrin on GLP-1 secretion in NCI-H716 cells}

Inoculation was performed with $5 \times 10^{4}$ NCI-H716 cells. We then measured the cell quantity and GLP-1 secretion every $24 \mathrm{~h}$. The results showed that the level of GLP-1 secretion significantly increased after the cell density reached $2 \times 10^{5} / \mathrm{mL}$, so isoquercitrin and sitagliptin stimulated the cells when the density was $2 \times 10^{5} / \mathrm{mL}$. It was found that isoquercitrin treatment may increase GLP-1 secretion in a concentrationdependent manner (Fig. 2A), as determined using ELISA, and the level of GLP-1 significantly increases beginning at $6 \mathrm{~h}(P<$ 0.01 ), when the GLP-1 level increased from the basal level of $5.73 \mathrm{pmol} \mathrm{L}^{-1}$ protein to $8.37 \mathrm{pmol} \mathrm{L}^{-1}$ protein.

Furthermore, we also evaluated the GLP-1 release in a positive control. In this study, we employed the well-known sitagliptin as a positive control. The results indicated that sitagliptin dose-dependently increased the level of GLP-1 (Fig. 2B).

Isoquercitrin decreases blood glucose levels and ameliorates body weight disorder in T2DM mice

Isoquercitrin affects the appearance and ameliorates the body weight disorder in T2DM mice. At the beginning of the study, we established a T2DM mouse model by high-fat feeding and treatment with low-dose STZ. The FBG of mice reached $11.1 \mathrm{mmol} \mathrm{L}^{-1}$, whereas the TG had no significant change (Fig. 4C and D). These results confirmed the successful

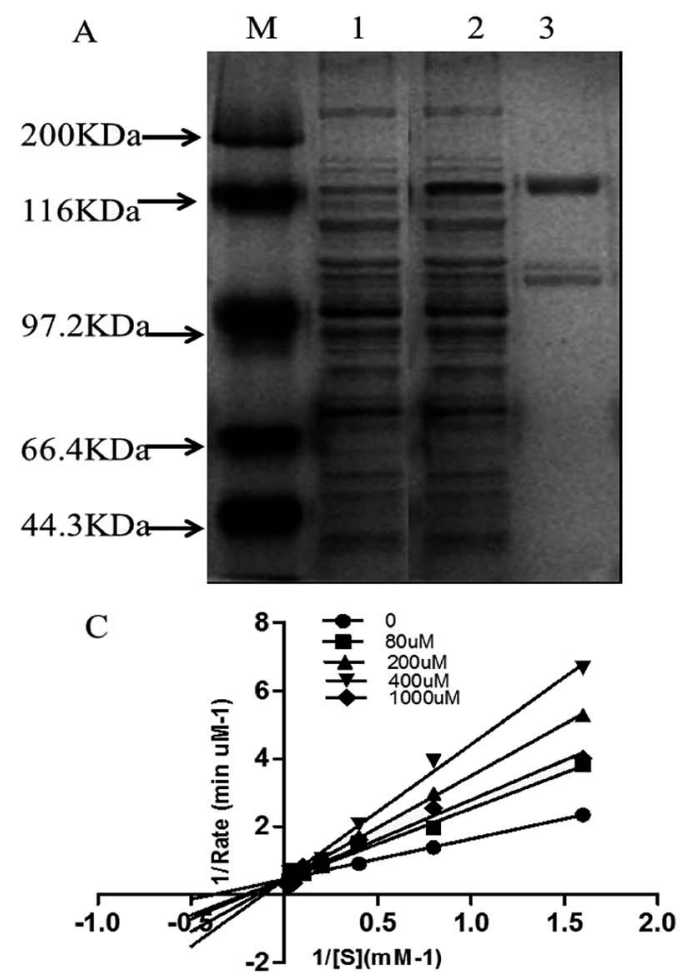

B

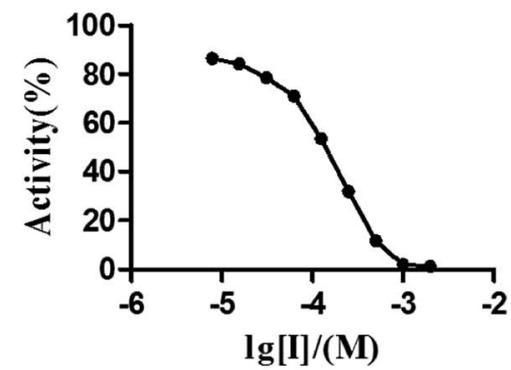

Fig. 1 DPP-IV (A) expression and purification of DPP-IV. (lane A: marker; lane 1: crude extraction; lane 2: after induction; lane 3: after purification.) (B) IC 50 value of isoquercitrin against DPP-IV; (C) inhibition type of isoquercitrin against $\triangle D P P-I V$; (D) inhibition constant of isoquercitrin against $\triangle \mathrm{DPP}-\mathrm{IV}$. 

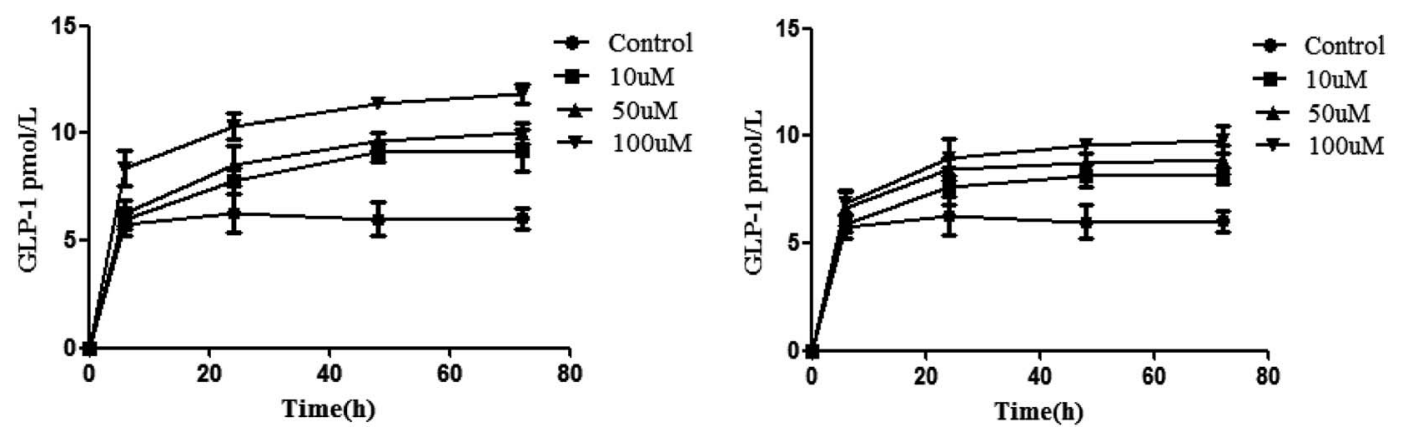

Fig. 2 GLP-1 secretion in $\mathrm{NCl}-\mathrm{H} 716$ cells. (Isoquercitrin; sitagliptin).

establishment of the T2DM model. It was very obvious that the model mice with polydipsia and polyuria lost body weight; the water consumption in the model group was greater than that in the normal group, the hair was extremely dull, and the spirit was listless. The condition of the isoquercitrin group was better than that of the model group.

The body weight stably increased in the normal mice but decreased in the model mice (Fig. 3A). The value in the isoquercitrin-treated mice and sitagliptin-treated mice decreased during the early treatment and then gradually increased, and there were not an obvious difference compared to the normal group. The weight of the mice in the isoquercitrin high dose group and sitagliptin group were higher than that in the low dose group, and the change occurred in a dosedependent manner.

The body weight significant decreased in diabetic model mice after 8 weeks, whereas the weight increased in the other groups of mice. The body weight of the mice in the isoquercitrin group and the sitagliptin group were higher than that before treatment $(P<0.001)$. The body weight exhibited no statistically significant difference at the end of treatment in the isoquercitrin-treated mice compared with normal mice and sitagliptin-treated mice. These results suggest that isoquercitrin can ameliorate body weight disorder in T2DM mice.

\section{Isoquercitrin decreases the blood glucose levels and glucose tolerance of T2DM mice}

We administered isoquercitrin, sitagliptin or vehicle for 8 weeks to these mice. The FBG in the diabetic model mice gradually increased and was significantly higher than that in the normal mice (Fig. 3B). The blood glucose level increased significantly in the diabetic model mice after 8 weeks. At the end of the 8 weeks of treatment, the blood glucose level in the high dose isoquercitrin-treated mice and the sitagliptin group was significantly decreased compared with that in the diabetic mice $(P<0.001)$. However the blood glucose levels of the low-dose group and medium-dose group mice declined slightly. These results indicated that isoquercitrin was an efficient hypoglycemic reagent, although further in vivo experiments,
A

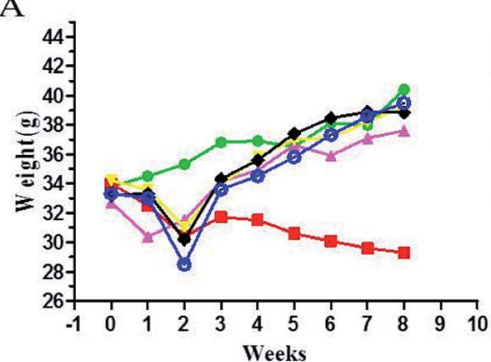

$\mathrm{C}$

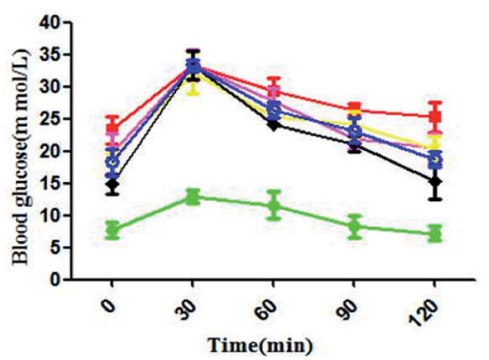

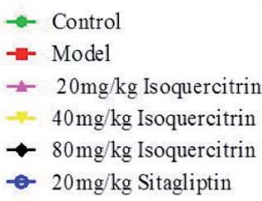

- Normal

- - Model

- $20 \mathrm{mg} / \mathrm{kg}$ Isoquercitrin

$40 \mathrm{mg} / \mathrm{kg}$ Isoquercitrin

- $80 \mathrm{mg} / \mathrm{kg}$ Isoquercitrin

- $20 \mathrm{mg} / \mathrm{kg}$ Sitagliptin
B

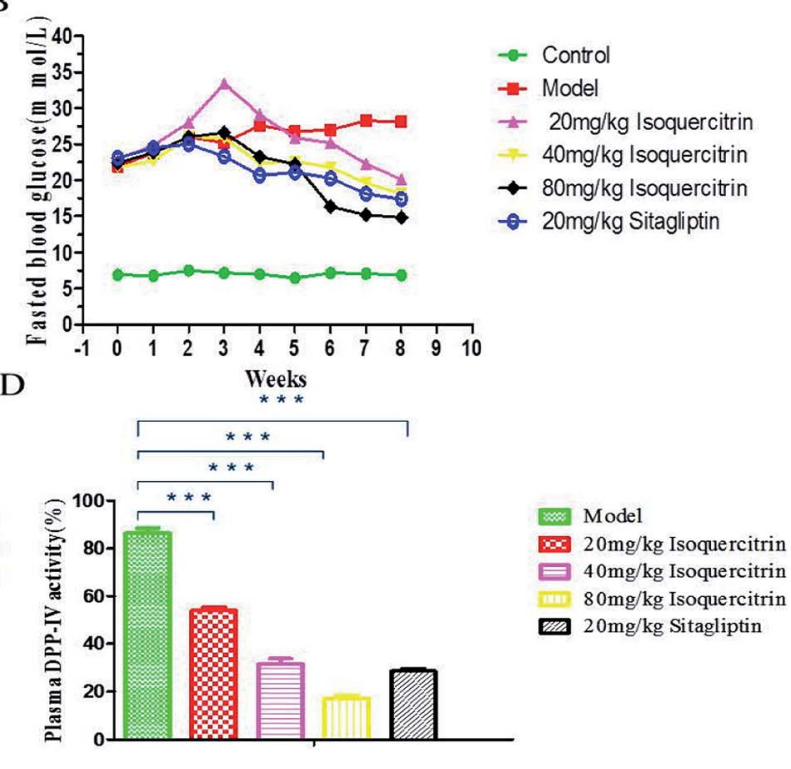

Fig. 3 Effect of isoquercitrin on body weight, blood glucose and DPP-IV activity in T2DM mice. ((A) effects of drugs on the body weight; (B) effects of drugs on blood glucose levels; (C) OGTT results; (D) plasma $\triangle$ DPP-IV activity of mice.*** $P<0.001 ; * * P<0.01$; *P<0.05). 
particularly with various dosages, are required to better estimate its therapeutic potential. A OGTT showed that the blood glucose concentration significantly decreased in the treatment group compared with that in the model group. The mediumdose and high-dose isoquercitrin treatment and sitagliptin obviously antagonized the blood glucose rise caused by exogenous glucose, and low doses of isoquercitrin also reduced the blood glucose level (Fig. 3C).

\section{Effects of isoquercitrin treatment on the DPP-IV activity, GLP- 1 level and insulin level in serum}

To address how isoquercitrin exerts its effect on the amelioration of hyperglycaemia in T2DM mice, we investigated the DPPIV activity and GLP-1 secretion levels in the serum. The DPP-IV activity in serum rapidly decreased in the isoquercitrin- and sitagliptin-treated diabetic mice. Different concentrations of isoquercitrin have an obvious inhibitory effect in the serum of DPP-IV in mice treated for 8 weeks, which showed the obvious dose-dependent increase in the inhibitory effect of DPP-IV (Fig. 3D).

The GLP-1 levels in portal plasma were investigated after a glucose load (Fig. 4A), and the area under concentration-time curves from 0 to 30 min was estimated using the linear trapezoidal rule. It was found that a glucose load might induce GLP-1 release. The serum levels of GLP-1 of the mice in the isoquercitrin group and the sitagliptin group were higher than those in the model group $(P<0.001)$. The high dose of isoquercitrin $\left(80 \mathrm{mg} \mathrm{kg}^{-1}\right)$ may enhance the GLP-1 release induced by the glucose load, and the peak level occurred at $10 \mathrm{~min}$. The GLP-1 level rose from 1.04 to $1.3 \mathrm{pmol} \mathrm{L}^{-1}$ in model group mice and increased obviously in the high dose group mice, with the level increasing from 1.15 to $1.72 \mathrm{pmol} \mathrm{L}^{-1}(N=10)$. The level of serum GLP-1 was decreased in all of groups at $20 \mathrm{~min}$. However, the level almost decreased to the original level after $30 \mathrm{~min}$. The GLP-1 level of the mice in model group increased slightly after glucose stimulation, which may result from the high DPP-IV activity in vivo, and GLP-1 was degraded.

\section{Effects of isoquercitrin treatment on insulin levels}

Plasma insulin levels were measured following a glucose load (Fig. 4B). It was found that a glucose load might induce an increase in the insulin level in plasma, and the peak concentration occurred at $20 \mathrm{~min}$. The plasma insulin level significantly increased in a dose-dependent manner, with the value increasing from $4.6 \mathrm{mIU} \mathrm{L}^{-1}$ to $7.4 \mathrm{mIU} \mathrm{L}^{-1}$ in the high-dose isoquercitrin group $(N=10)$. The level of insulin decreased in all groups at $30 \mathrm{~min}$. However, the level almost decreased to the original level after $120 \mathrm{~min}$. The levels of serum insulin in the mice of the isoquercitrin group and the sitagliptin group were higher than those in the model group at the same time, especially in the high-dose isoquercitrin group $(P<0.001)$. Although treatment with the low dose of isoquercitrin tended to decrease the insulin level, the change was not significant.

\section{TC and TG characteristics of the lipid profile in the serum}

The TC in serum was significantly higher after T2DM inducement than before inducement, whereas TG did not increase. The pre- and post-treatment TC did not differ upon isoquercitrin or sitagliptin treatment (Fig. 4C). The TG in serum was lower after treatment than before treatment, but the difference was not significant (Fig. 4D).
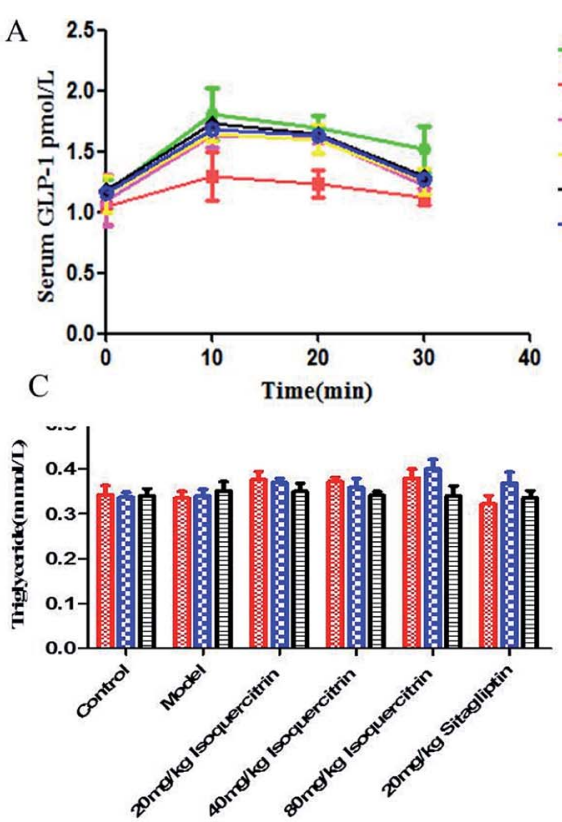
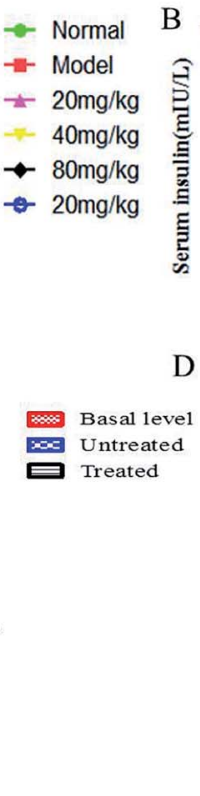
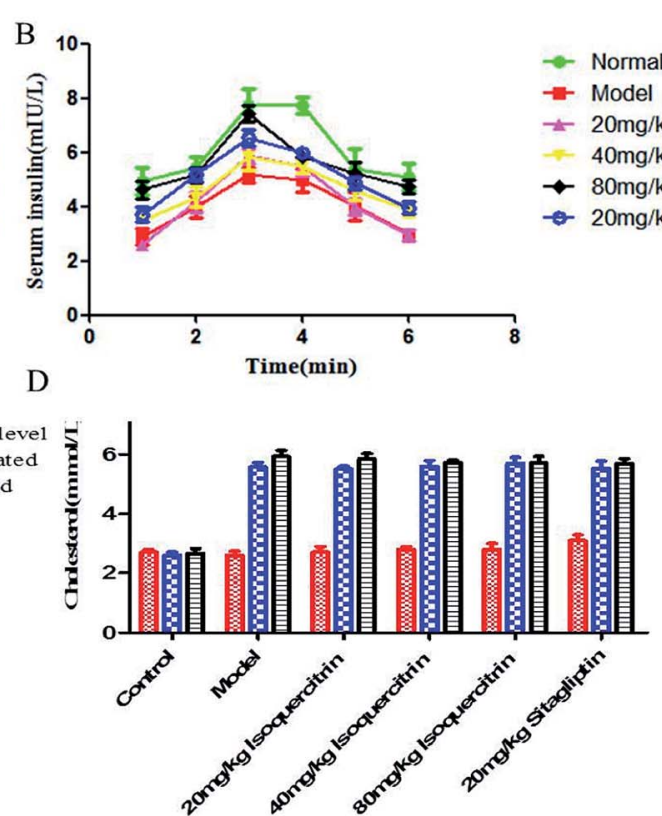

- Normal

-들 Model

$₫ 20 \mathrm{mg} / \mathrm{kg}$ Isoquercitrin

- $40 \mathrm{mg} / \mathrm{kg}$ Isoquercitrin

$\curvearrowleft 80 \mathrm{mg} / \mathrm{kg}$ Isoquercitrin

- 20mg/kg Sitagliptin

Fig. 4 Effect of isoquercitrin on GLP-1, insulin, TC and TG levels in T2DM mice. (A) Effects of drugs on GLP-1; (B) effects of drugs on the insulin level at different times; (C) triglyceride; (D) cholesterol level (B). 


\section{Discussion}

T2DM is characterized by increased levels of blood glucose due to impaired insulin sensitivity (insulin resistance). This study revealed several essential findings relevant to the hypoglycemic effect of isoquercitrin and proposed a new underlying mechanism, independent of the findings in previous studies. Therefore, the advised inhibition of DPP-IV activity is considered to evoke multiple sets of physiological responses to resist T2DM and obesity via facilitating tyrosine phosphorylation of insulin signalling molecules. In fact, several studies have reported that oral administration of a DPP-IV inhibitor can decrease glucose levels in T2DM mice. ${ }^{8-11}$

Pharmacological approaches for treating T2DM and obesity have focused partly on targeting the glucagon-like peptide- 1 (GLP-1) system..$^{12-15}$ GLP-1 is an incretin hormone produced in the L cells of the intestine that acts at the GLP-1 receptor (GLP1R) to improve glycaemic control, reduce food intake, and improve insulin sensitivity. ${ }^{16}$ Studies demonstrated that the role of GLP-1 in stimulating insulin secretion is significantly reduced in type 2 diabetes, which has been attributed to a decrease in GLP-1 receptor expression. ${ }^{17}$ Because native GLP-1 is rapidly degraded by the enzyme dipeptidyl peptidase-IV (DPPIV) ${ }^{18,19}$ pharmacological strategies include inhibiting DPP-IV activity and creating a GLP-1R agonist resistant to enzymatic degradation. Thus, DPP-IV could be one of the first candidates for the target molecule of isoquercitrin through which isoquercitrin acts to improve insulin sensitivity. As our findings indicated, isoquercitrin competitively inhibited DPP-IV activity in vitro by directly blocking the DPP-IV active-site pocket.

NCI-H716 cells served as in vitro models of intestinal L cell in further investigations of the effect of isoquercitrin in regulating GLP-1 release. Reports indicated that L cells secrete GLP-1 in response to stimulating the intestines, the concentration of GLP-1 depends on the islet beta cell expression of the GLP-1 receptor, and insulin secretion reduced the production of liver glycogen.$^{20} \mathrm{~A}$ study on the hypoglycemic effect of berberine also found that berberine decreased blood glucose by increasing the secretion of GLP-1. ${ }^{21}$ An in vitro study demonstrated that isoquercitrin may stimulate GLP-1 secretion from NCI-H716 cells in a dose-dependent manner; this change was accompanied by an increased GLP-1 level, which indicated that isoquercitrin may increase GLP-1 release in addition to promoting GLP-1 biosynthesis.

It was obvious that treatment through oral gavage with different concentrations of isoquercitrin resulted in a profound attenuation in hyperglycaemia. In particular, the strength of this hypoglycemic effect was comparable to that of sitagliptin (clinical drug). The anti-hyperglycaemic effect of sitagliptin, an antidiabetic drug, is due to the suppression of DPP-IV activity, which is same mechanism as for isoquercitrin, as we demonstrated. However, our findings in this study are the first, to the best of our knowledge, to demonstrate that isoquercitrin inhibits DPP-IV in vitro and in vivo, resulting in enhanced insulin secretion.
For type 2 diabetic patients, hyperglycaemia is often accompanied by dyslipidemia, which manifests as abnormal serum lipid profiles, such as TG and TC changes. Abnormal values of these indices result in a series of metabolic disorders and complications. This study indicated that the effect of isoquercitrin on serum TC and TG for the studied mice was not statistically significant. However, we found that isoquercitrin can alleviate bodyweight disorders, implying some multi-target mechanisms remain to be determined.

Here, we demonstrated that oral treatment with isoquercitrin resulted in a profound attenuation in hyperglycaemia in diabetic mice. The mechanism may be associated with the DPP-IV activity inhibition by isoquercitrin, but more studies are needed. An inhibitor of DPP-IV could stimulate the secretion of insulin by improving the activity, which significantly improved symptoms in type 2 diabetes. $^{22}$ The level of GLP-1 in plasma is associated with the activity of DPP-IV. A study showed that DA1229 , an inhibitor of DPP-IV, had an obvious inhibitory effect in vivo. Treatment with $300 \mathrm{mg} \mathrm{kg}^{-1}$ day $^{-1}$ DA-1229 through oral gavage resulted in an obvious inhibitory effect in the mouse serum, which is similar to our results. ${ }^{23}$ Studies suggested that different quercetin reduced the blood sugar in type 2 diabetes mice by inhibiting $\alpha$-glycosidase enzyme activity, with the effect depending on whether the tested dose was $50 \mathrm{mg} \mathrm{kg}^{-1}$ or $200 \mathrm{mg} \mathrm{kg}{ }^{-1} \cdot{ }^{24}$ However, our findings in this study are the first, to the best of our knowledge, to demonstrate that isoquercitrin inhibits DPP-IV in vitro and in vivo, resulting in enhanced GLP-1 and insulin secretion and then amelioration of hyperglycaemia in high-fat diet and STZ-induced T2DM mice.

In vivo studies showed that isoquercitrin treatment might lower the fasting blood glucose level, and this change is accompanied by an increase of insulin levels in plasma. Treatment with $80 \mathrm{mg} \mathrm{kg}^{-1}$ isoquercitrin might significantly increase the GLP-1 release induced by a glucose load, with this change accompanied by a higher GLP-1 level in portal plasma. Peripherally released GLP-1 enters brain areas and participates in the regulation of the anorexic response, ${ }^{25}$ which indicates that the suppression of food intake induced by isoquercitrin might partly lead to the enhancement in GLP-1 release. It is wellknown that GLP-1 exerts important effects on regulating glucose homeostasis via stimulating insulin secretion, beta-cell proliferation, inhibiting food intake. ${ }^{26}$ All of these results further supported our previous findings that isoquercitrin exerted its antidiabetic effects partly via increasing GLP-1 release. These findings further indicated the possibility of enhancement of GLP-1 biosynthesis by isoquercitrin. ${ }^{27,28} \mathrm{~A}$ study showed that the insulin secretion stimulated by GLP-1 in vivo was consistent with that found in the OGTT, which was consistent with our experimental results.

Although we have proved the hyperglycaemic effects of isoquercitrin on the type 2 diabetes mice occur by targeting DPPIV, there are also some limitations for this study. First, there are many other pathways affecting type 2 diabetes other than the inhibition of DPP-IV. Second, there may be several other substrates of DPP-IV in cells, which may have some adverse effects on the complete or partial inhibition of DPP-IV. 
Therefore, if applying isoquercitrin into clinical therapy, the side effects of isoquercitrin must be investigated in future studies.

\section{Conflicts of interest}

The authors declare that there is no conflict of interests regarding the publication of this paper.

\section{Acknowledgements}

This work was supported by the previous research project of Mudanjiang Normal University [grant number SY2014009] and the education department record project of Heilongjiang [grant number 1352MSYQN002]. The first author of this paper is now working at Mudanjiang Normal University at Heilongjiang University of China. The author wishes to thank Dr Xue-Qi Fu for his kind help. All animal procedures were performed in accordance with the Guidelines for Care and Use of Laboratory Animals of Jilin University and approved by the Animal Ethics Committee of Department of Science and Technology of Jilin Province.

\section{References}

1 R. A. DeFronzo, From the triumvirate to the ominous octet: a new paradigm for the treatment of type 2 diabetes mellitus, Diabetes, 2009, 58, 773-795.

2 A. Wang, C. Dorso, L. Kopcho, G. Locke, R. Langish, E. Harstad, P. Shipkova, J. Marcinkeviciene, L. Hamann and M. S. Kirby, Potency, selectivity and prolonged binding of saxagliptin to DPP4: maintenance of DPP4 inhibition by saxagliptin in vitro and ex vivo when compared to a rapidlydissociating DPP4 inhibitor, BMC Pharmacol., 2012, 12(4), 2.

3 C. F. Deacon, E. Mannucci and B. Ahrén, Glycemic efficacy of GLP-1 receptor agonists and DPP-4 inhibitors as addon therapy to metformin in subjects with type 2 diabetes a review and meta analysis, Diabetes, Obes. Metab., 2012, 14(8), 762-767.

4 N. González-Abuín, N. Martínez-Micaelo, M. Blay, G. Pujadas, S. Garcia-Vallvé, M. Pinent and A. Ardévol, Grape seed-derived procyanidins decrease dipeptidylpeptidase 4 activity and expression, J. Agric. Food Chem., 2012, 60(36), 9055-9061.

5 P. Bansal, P. Paul, J. Mudgal, P. G. Nayak, S. T. Pannakal, K. I. Priyadarsini and M. K. Unnikrishnan, Antidiabetic, antihyperlipidemic and antioxidant effects of the flavonoid rich fraction of Pilea microphylla (L.) in high fat diet/ streptozotocin-induced diabetes in mice, Exp. Toxicol. Pathol., 2012, 64(6), 651-658.

6 V. Buttereweck and M. Hegger, Winterhoff H. Flavonoids of St. John's Wort reduce HPA axis function in the rat, Planta Med., 2004, 70(10), 1008.

7 Q. Liu, R. Bonneville, T. Li and V. X. Jin, Transcription factorassociated combinatorial epigenetic pattern reveals higher transcriptional activity of TCF7L2-regulated intragenic enhancers, BMC Genomics, 2017, 18(1), 375.
8 A. J. Scheen and N. Paquot, About the choice between a DPP4 inhibitor and a SGLT 2 inhibitor tor treating type 2 diabetes, Rev. Med. Liege, 2016, 71(12), 579-585.

9 B. AM, L. M. Real Hernandez, M. A. Berhow and E. G. de Mejia, Bioactive compounds from culinary herbs inhibit a molecular target for type 2 diabetes management, dipeptidyl peptidase IV, J. Agric. Food Chem., 2014, 62(26), 6147-6158.

10 Y. Sun and L. Ma, Role of dipeptidyl peptidase- 4 and its inhibitor in the respiratory diseases, Zhongnan Daxue Xuebao, Yixueban, 2017, 42(1), 108-112.

11 H. Guo, C. Fang, Y. Huang, Y. Pei, L. Chen and J. Hu, The efficacy and safety of DPP4 inhibitors in patients with type 1 diabetes: A systematic review and meta-analysis, Diabetes Res. Clin. Pract., 2016, 121, 184-191.

12 S. A. Sadry and D. J. Drucker, Emerging combinatorial hormone therapies for the treatment of obesity and T2DM, Nat. Rev. Endocrinol., 2013, 9, 425-433.

13 S. Chatterjee, S. Ghosal and S. Chatterjee, Glucagon-like peptide-1 receptor agonists favorably address all components of metabolic syndrome, World J. Diabetes, 2016, 7, 441-448.

14 R. Burcelin and P. Gourdy, Harnessing glucagon-like peptide-1 receptor agonists for the pharmacological treatment of overweight and obesity, Obes. Rev., 2017, 18(1), 86-98.

15 S. P. Rajeev and J. Wilding, GLP-1 as a target for therapeutic intervention, Curr. Opin. Pharmacol., 2016, 31, 44-49.

16 J. J. Holst, The physiology of glucagon-like peptide 1, Physiol. Rev., 2007, 87, 1409-1439.

17 A. Y. Y. Mizokami, J. Gao, M. Matsuda, I. Takahashi, H. Takeuchi and M. Hirata, Osteocalcin induces release of glucagon-like peptide-1 and thereby stimulates insulin secretion in mice, PLoS One, 2013, 8(2), 1-8.

18 M. Zander, S. Madsbad, J. L. Madsen, et al., Effect of 6-week course of glucagon-like peptide 1 on glycaemic control, insulin sensitivity, and beta-cell function in type 2 diabetes: A parallel-group study, Lancet, 2002, 359(9309), 824-830.

19 R. Mentlein, Dipeptidyl-peptidase IV (CD26)-role in the inactivation of regulatory peptides, Regul. Pept., 1999, 85, 9-24.

20 A. Wettergren, M. Wojdemann and J. J. Holst, The inhibitory effect of glucagon-like peptide-1 (7-36)amide on antral motility is antagonized by its n-terminally truncated primary metabolite glp-1 (9-36)amide, Peptides, 1998, 19(5), 877-882.

21 Y. Yu, L. Liu, X. Wang, et al., Modulation of glucagon-like peptide-1 release by berberine: In vivo and in vitro studies, Biochem. Pharmacol., 2010, 79(7), 1000-1006.

22 J. Mu, A. Petrov, G. J. Eiermann, et al., Inhibition of dpp-4 with sitagliptin improves glycemic control and restores islet cell mass and function in a rodent model of type 2 diabetes, Eur. J. Pharmacol., 2009, 623(1-3), 148-154.

23 M. K. Kim, Y. N. Chae, H. D. Kim, et al., Da-1229, a novel and potent dpp4 inhibitor, improves insulin resistance and delays the onset of diabetes, Life Sci., 2012, 90(1-2), 21-29. 
24 Z. Rui and W. Yingping, Study on Tartary Buckwheat Flavonoids and its Antidiabetes Activity, Chinese Academy of Agricultural Sciences, 2008, 6, 25-28.

25 C. Orskov, S. S. Poulsen, M. Moller and J. J. Holst, Glucagonlike peptide-1 receptors inthe subfornical organ and the area postrema are accessible to circulating glucagon-like peptid1, Diabetes, 1996, 45, 832-835.

26 L. R. Ranganath, Incretins: pathophysiological and therapeutic implications of glucose-dependent insulinotropic polypeptide and glucagon-like peptide-1, Clin. Pathol., 2008, 61, 401-409.
27 S. S. Lu, Y. L. Yu, H. J. Zhu, X. D. Liu, L. Liu, Y. W. Liu, et al., Berberine promotes glucagon-like peptide-1 (7-36) amide secretion in streptozotocin-induced diabetic rats, $J$. Endocrinol., 2009, 200, 159-165.

28 M. Laakso, J. Zilinskaite, T. Hansen, et al., Insulin sensitivity, insulin release and glucagon-like peptide-1 levels in persons with impaired fasting glucose and/or impaired glucose tolerance in the eugene2 study, Diabetologia, 2008, 51(3), 502-511. 